\title{
Foot and Mouth Disease Vaccine Development and Challenges in Inducing Long-Lasting Immunity: Trends and Current Perspectives
}

\author{
Ambaye Kenubih (D) \\ University of Gondar, College of \\ Veterinary Medicine and Animal Sciences, \\ Para-Clinical Studies, Gondar, Ethiopia
}

\begin{abstract}
Foot and mouth disease (FMD) is an extremely contagious viral disease of livestock caused by foot and mouse disease virus genus: Aphthovirus, which causes a serious economic impact on both individual farmers and the national economy. Many attempts to advance a vaccine for FMD have failed to induce sterile immunity. The classical methods of vaccine production were due to selective accumulation of mutations around antigenic and binding sites. Reversion of the agent by positive selection and quasi-species swarm, use of this method is inapplicable for use in non-endemic areas. Chemical attenuation using binary ethyleneimine (BEI) protected the capsid integrity and produced a pronounced immunity against the challenge strain. Viral antigens which have been chemically synthesized or expressed in viruses, plasmid, or plants were tried in the vaccination of animals. DNA vaccines expressing either structural or nonstructural protein antigens have been tried to immunize animals. Using interleukins as a genetic adjuvant for DNA vaccines have a promising effect. While the challenges of inducing sterile immunity lies on nonstructural (NS) proteins of FMDV which are responsible for apoptosis of dendritic cells and have negative effects on lympho-proliferative responses which lead to transient immunosuppression. Furthermore, destruction of host protein trafficking by nonstructural proteins suppressed $\mathrm{CD}_{8}{ }^{+} \mathrm{T}$-cell proliferation. In this review, it tried to address multiple approaches for vaccine development trials and bottle necks of producing sterile immunity.
\end{abstract}

Keywords: FMD vaccine, long lasting immunity, sterile immunity

\section{Background}

Foot and mouth disease (FMD) is an extremely contagious viral infection of livestock caused by Foot and Mouse disease virus, genus: Aphthovirus and family Picornaviridae; that inflicts severe economic losses. ${ }^{1,2}$ Domestic and wild ungulates are highly affected by FMD. ${ }^{3-5}$ The $5 \mathrm{~S}$ protomers are spontaneously produced by individual mature proteins; VP1, VP3, and VP0, five of which assemble into a $12 \mathrm{~S}$ pentamer. While the $75 \mathrm{~S}$ viral capsid is formed by the assembly of the 12 pentamers, ${ }^{6,7}$ the complete foot and mouth disease virus (FMDV) 146S antigen has been reported to have very similar antigen specificity to the viral capsid., ${ }^{8,9}$ Foot-and-mouth disease virus has a wide host range, a high rate of genetic variation, and great antigenic differences, and it has seven serotypes (A, O, C, Asial, SAT1, SAT2, and SAT3) and more than 100 serosubtypes. ${ }^{10}$ Due to quasi species swarm, many new variants also appear every year. There is no cross-immunity induced by the seven serotypes. There is also only a partial cross-immunity between
Correspondence: Ambaye Kenubih Email ambayken@yahoo.com 
the various subtypes of the same serotype. ${ }^{11}$ The variability in and polymorphism of FMDV have made the prevention and control of FMD very difficult. ${ }^{10}$ Adenovirus recombinant FMDV expressing $\mathrm{P} 12 \mathrm{~A}$ and $3 \mathrm{C}$ proteins of different serotypes have shown a protective effect. ${ }^{12-14}$

Classical inactivated vaccines have many shortcomings, including thermal instability, short lived immunity, high cost, risk of recombination with the wild strains, and reversion of the pathogensity. ${ }^{11,15,16}$

Despite 90 years of research, there is no effective vaccine that produces sterile and solid immunity for FMD, but the disease remains enzootic in large areas of the globe. Many attempts to develop a vaccine against FMD have failed to induce sterile immunity, with little cross-serotype protection, and inadequate duration of immunity. ${ }^{17}$ The classical methods of vaccine production, like serial passage of the virus on cell culture, ${ }^{18}$ in nonpermissive animals and in its own natural host were able to attenuate the virus. This is due to the selective accumulation of mutations around antigenic and binding sites. ${ }^{19-21}$ However, use of the reversion of the agent by positive selection and quasi-species swarm method is inapplicable for use in non-endemic areas. ${ }^{22}$ DNA and protein technologies have improved the research by modifying integrin receptors, ${ }^{23}$ utilizing synthetic peptides that can be able to induce an explicit immune response in the animal. ${ }^{24}$ T-helper cell epitopes intrinsic to the $\mathrm{G}-\mathrm{H}$ loop region are potent B-cell epitopes that induce humoral immunity. ${ }^{25}$ The use of recombinant interferon $\alpha$ (IFN $\alpha$ ) and bovine interleukin 18 (IL-18) as an adjuvant have enhanced long-term immunity in laboratory animals. ${ }^{26,27}$

Recently, recombinant live vectored DNA vaccines have been found to be potent cytotoxic $\mathrm{T}$ lymphocytes (CTL) inducers, ${ }^{28,29}$ in order to pronounce this effect in FMD an appropriate adjuvant is necessary. ${ }^{30}$ To overcome this effect, recombining the host Ig super family and FMD epitope have improved the humoral and cellular immune response of the animal.

The bottle neck problem in inducing sterile and longlasting immunity is hampered by destruction of the host protein trafficking by nonstructural (NS) proteins, specially $3 \mathrm{~A}$, so does not elicit a cluster differentiation 8 positive $\left(\mathrm{CD}_{8}{ }^{+}\right)$T-cell response due to weak $\mathrm{CTL}$ and the virus persists in the animal. ${ }^{31}$ Integrin receptors which mediated bone marrow-derived dendritic cell (DC) apoptosis hindered the innate immunity of the host. ${ }^{32}$ Guzman et $\mathrm{al}^{33}$ and Joshi et $\mathrm{al}^{34}$ analyzed surrogate responses for CTL killing such as proliferation or production of IFN $\gamma$ by $\mathrm{CD}_{8}$ expressing cells, but many $\mathrm{CD}_{8}{ }^{+}$expressing lymphocytes that are not CTLs produce IFN $\gamma$, including natural killer (NK) cells and subsets of gamma delta $(\gamma \delta)$ T-cells. $^{35-37}$

According to Oh et al, ${ }^{38}$ IFN- $\gamma$ response can be restimulated in vaccinated cattle who have shown a high level of virus neutralizing antibody titer in the circulation on the day of challenge, which has a direct relation to vaccine-induced protection with IFN- $\gamma$ and neutralizing antibody. Furthermore, the $\mathrm{CD}_{4}{ }^{+} \mathrm{T}$-cells are the main flourishing phenotype and IFN- $\gamma$ producing cells. However, in natural infection there lymphopenia, which overlapped with peak viremia and serum IFN- $\alpha$ response while in vivo plasmocyte $\mathrm{DC}(\mathrm{pDC})$ numbers and in vitro pDC IFN- $\alpha$ secretion briefly declined within 2 days of infection. ${ }^{39}$ Production of IFN- $\alpha$ from monocyte-derived DCs (MoDCs) and skin-derived DCs (skin DCs) is inhibited in the acute phase of infection of swine. ${ }^{40}$ There was also induction of apoptosis in immature dendritic cells by FMDV. $^{32}$ To overcome the immune pathogenesis of the virus by boosting antibody production and T-cell proliferation, higher levels of CTL activity and IFN- $\gamma$ expression in $\mathrm{CD}_{8}$ T-cells were achieved by synthetic oligonucleotides as mucosal vaccine adjuvants. ${ }^{41}$ The main aim of this paper is to review trends in FMD vaccine development and challenges to induce sterile and long-term immunity.

\section{Attenuated and Inactivated Vaccine}

There were many attempts to improve live attenuated FMD vaccines by the conventional methods like serial passage in non-permissive animals or in cell culture. Attenuation was achieved by passaging in nonsusceptible species, such as mice, rabbits, and embryonated eggs, until it lost virulence in cattle. Serial passages of FMDV C-S8c1 at high multiplicity of infection in cell culture resulted in a defective genome (C-S8p260), which was completely protective for mice against lethal challenge with FMDV C-S8c1 and safe for swine after vaccination with a single dose of C-S8p260. ${ }^{42}$ It also induced high titers of neutralizing antibodies and activated T-cells in swine. ${ }^{42}$

Serial contact transmission of the highly pathogenic, pig-adapted O Taiwan 97 isolate in swine significantly reduces the virulence after the 14th pig passage and abolished it after the 16th passage. ${ }^{43}$ Amino acid changes during in vivo passages were highly silent substitution and changes in VP1 (1D) were transitory. Developing FMD vaccine in non-permissive hosts may trigger the 
agent to use other cellular receptors other than Arg-GlyAsp (RGD), a plaque test in BHK 21 test might show as negative while the virus is intact. ${ }^{43}$ Replacement of the amino acid side chains located near the capsid intersubunit by another amino acid could establish new disulfide bonds or electrostatic interactions between subunit interfaces and is either projected or initiated to be expendable for infection by increasing heat tolerance of the vaccinal strains, ${ }^{44}$ using current procedures, of FMD vaccines that are less reliant on an ideal cold chain. FMDV C-S8p260 strains are segmented as well as replicationcompetent which can provide the basis of attenuation of vaccines with two safety barriers. ${ }^{45}$

Most research shown that animal model FMDV inactivation using an amino acid substitution in $2 \mathrm{C}$ was not detectable in C-S8c1 but was present in a low proportion of the guinea pig-adapted FMDV. ${ }^{46}$ This amino acid substitution became rapidly overriding in the viral population after the reintroduction of the guinea pig-adapted virus into pigs. These findings show how the introduction of minority variant viruses in an artificial host might rise to their dominance when the original host species is reinfected. ${ }^{47}$ In addition to this positive selection and quasi-species swarm, vaccinal strain may reverse to pathogenic strain. ${ }^{22}$

Inactivation of the virus by using formalin and endonuclease fails to inactivate the virus and degrade the antigenic sites, respectively. ${ }^{48}$ Binary ethyleneimine (BEI) has also been used for attenuation of the virus while keeping the integrity of the capsid. ${ }^{15,49}$ BEI-inactivated FMD cellular receptors, integrins, are used for attachment and internalization into cultured cells, interaction is mediated by an amino acid residue located within the G-H loop of VP1 capsid protein. ${ }^{49}$ FMDV-specific monoclonal antibody or a synthetic peptide showed binding of BEI-inactivated FMD were internalized by their co-localization with the marker protein into BHK-21, mediated by the integrinbinding motif RGD. ${ }^{50}$ In addition, BEI-inactivation has no effect on the antigenicity of the G-H loop. ${ }^{20,51}$ BEI inactivation, preserved virion architecture and receptors favored the internalization of the virus into cultured cells, as well as in vivo, is mediated by integrin recognition, ${ }^{47,52,53}$ however, whole virus quantification shows a minimum result compared to that of formalin inactivation (65-71.6\%), while BEI inactivation is $44.2 \%{ }^{49}$

Sequential passaging of type C FMDV from swine and guinea pigs, and maintained in swine and suckling mice, results in amino acid replacements (I2483T in 2C, Q443R in 3A, and L1473P in VP1). The replaced amino acid (L1473P), next to the integrin-binding RGD motif, abolished growth of the virus in different cell lines and altered its antigenicity. ${ }^{47}$ Another study conducted by Burman et $\mathrm{al}^{20}$ revealed that a single amino acid change in the RGD+1 and RGD+4 sites inhibits virus attachment and infection facilitated by $\alpha v \beta 6$ or $\alpha v \beta 8$, but the virus utilizes $\alpha v \beta 3$ for cell attachment. Replacements with methionine or arginine at the binding site are effective inhibitors for $\alpha v \beta 6$. Two leucine residues at spots RGD +1 and RGD+4 stabilized the binding reliant on the structure immediately $\mathrm{C}$ terminal to the $\mathrm{RGD} .^{20,54}$ EDTA-resistant binding to $\alpha v \beta 6$ is a hallmark, and the stable complex with its cellular receptor was anticipated to produce considerable FMDV high infectiousness. ${ }^{21}$

Alanine substitution with leucines at the first and fourth positions at the RGD +1 and $\mathrm{RGD}+4$ sites results in inhibition of virus binding and virus attachment to $\alpha \mathrm{v} \beta 6$ or $\alpha \mathrm{v} \beta 8$. However, the virus utilizes $\alpha v \beta 3$ for cell entry. ${ }^{20}$ Whereas cell culture adapted strains use the heparan sulphate proteoglycans (HSPG) as an alternative receptor. The conformation of their ectodomains and the ligand-binding state of integrins is an important factor for tropism for viruses. ${ }^{54}$

Heparan sulphate binding viruses internalized DC effectively but did not present antigen to lymphocytes, inducing an FMDV-specific IgG response. ${ }^{55}$ These results demonstrate that DC internalization of FMDV is most efficient for vaccine viruses with HS-binding capacity, but HS binding is not an exclusive requirement.

Selective pressure exerted by the host humoral immune response has an important role in both selection and stabilizing of the antigenic FMDV variants, which results in alteration in cell tropism. In vitro tests revealed that parallel passage of FMDV in the presence of sub-neutralizing homologous sera resulted in the maintenance of mutants. ${ }^{56}$ However, propagation of FMD type A24 which contained an SGD sequence in the cell receptor-binding site were inoculated in cattle, the virus grew poorly in BHK-21 cells and the sequence were stably maintained during propagation in BHK-21 cells expressing the bovine $\alpha \mathrm{V} \beta 6$ integrin (BHK3 $\alpha \mathrm{V} \beta 6$ ), as well as in experimentally inoculated and contact cattle. $^{56}$

From two independent transmission chains in cattle, there are genomic changes due to sequential passage on BHK-21-cell-adapted (heparan sulphate binding) strain of FMDV. A wild-type variant with an amino acid mutation at VP1 356 was rapidly picked for in vivo viral replication. ${ }^{57}$

The deletion genes encode the NS protein that is not crucial for virus replication in vitro is an alternative 
technique to generate live attenuated vaccines. However, to be useful as a vaccine, this deletion virus must still be able to replicate in susceptible animals. The advantage of this approach, compared to the classical method of attenuation, which generally introduces mutations at a limited number of sites, is that the risk of reversion to virulence is significantly reduced $^{58}$ and NS proteins are potent T-cell epitopes. ${ }^{59}$

The effect has been shown in genetically modified FMDV vaccine strain with some amino acid replacement on the antigenic sites, with similar growth properties to the wild virus proven to fully protect the animal from challenge but with the ability replicate in vitro. ${ }^{60}$

Emergency double oil adjuvanted FMD vaccine showed a reduction in viraemia and virus shading, and showed no clinical signs. There was a consistent detection of IL-6, IL-8, and IL-12 in vaccinated animals. ${ }^{61}$ While other cytokines IL-1, IL-2, TNF, TGF, and interferons were not detected; this exhibits that the vaccine did not induce a systemic inflammatory response as well as systemic elevation of $\mathrm{T}$ lymphocyte activity, which is related to short lived protection against FMD. ${ }^{61}$

Recombinant human IL-2 is a potent humoral immune inducer in murine model FMD vaccine. ${ }^{62}$ Vaccinated animals remain seroconvert positive for 7-8 months and systemic levels of the cytokines (IL-6, IL-8, and IL-12) increased after vaccination. ${ }^{63}$

\section{Empty Viral Capsids}

Empty viral capsids, also known as virus like particles (VLP), comprise the entire repertoire of immunogenic sites found on intact viruses but lack infectious nucleic acids and involve the cloning of the viral genome essential for the synthesis, processing, and assembly of viral structural proteins into empty viral capsids (P1-2A and 3C ${ }^{\text {pro }}$ coding genes; Figure 1). ${ }^{64}$ Empty capsids are naturally produced in vitro in cell culture, are antigenically similar, and are immunogenic. ${ }^{65}$

The use of empty capsids vaccine are a promising candidate since it circumvents the use of virus in vaccine production and conserves the conformation of epitopes. ${ }^{45}$ Furthermore, there is no risk of reversion of the virus and recombination with the wild strains. Serum-free suspensiongrowing mammalian cells have been used for transient gene expression (TGE) of FMDV recombinant empty capsids. ${ }^{66}$ Identification of vaccinated from infected or convalescent animals is easy using currently available technology. ${ }^{67-69}$

\section{Subunit Vaccine}

Subunit vaccine contains viral antigens harvested by chemical extraction or bio-expression of a minimal amount of non-viral antigens in a culture medium. ${ }^{70}$ Investigators have revealed that VP1 is one of the FMDV capsid proteins, which had a significant surface exposure in the 1970s, and recent advances in structural virology. ${ }^{71}$

Genetic engineering has been used to mutate parts of the genome or abolish a protein-coding region of VP1 in recent attempts to create attenuated vaccines. Using recombinant DNA, a virus with the RGD receptor binding site on VP1 removed FMDV was constructed. ${ }^{72}$ In 7 - to 10 -day-old mice or pigs, this virus was unable to adhere to cells and did not induce infection. ${ }^{73}$

The VP1 capsid protein of FMDV and the carboxy terminal region of VP1 has a G-H loop which is highly

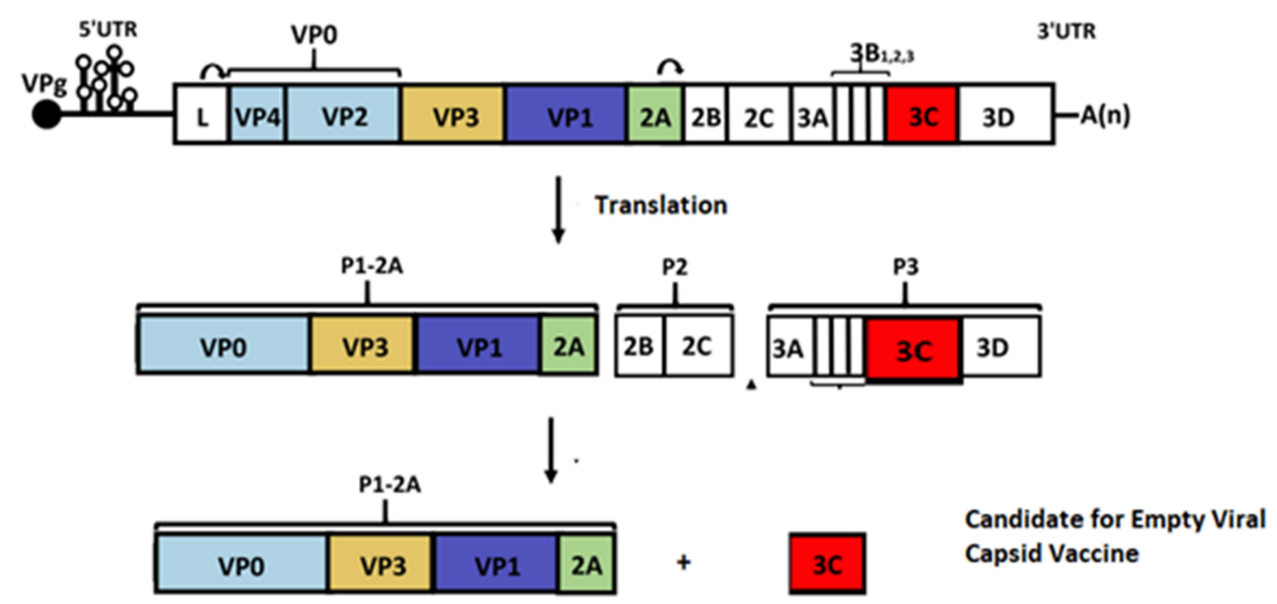

Figure I Schematic illustration of empty capsid vaccine production. The FMDV RNA gene (top) encodes a large polyprotein that is translated to a collection of precursors (PI-2A, P2, and P3) (middle). The bottom shows a candidate for empty viral capsid protein vaccine.

Note: Adapted from Belsham, GJ. Towards improvement in foot-and-mouth disease vaccine performance. Acta Vet Scand. 2020;62(I):20. http://doi.org/I0.I I86/s I3028-020$00519-1.105$ 
immunogenic, corresponding to the B-cell epitopes. A chemically synthesized peptide consisting of regions (residues 141 to 158 ) of a virus coating protein (VP1) from the FMDV serotype $\mathrm{O}$ have provoked high levels of neutralizing antibody and protected cattle against intradermolingual inoculation of infectious virus, ${ }^{24}$ suggesting the importance of the $\mathrm{G}-\mathrm{H}$ loop in inducing an humoral immune response. The VP1 also contains the hypervariable region and the immunogenic site; exploiting the site would be the base for broad immunogenicity. ${ }^{74}$

Incorporation of IFN as a genetic adjuvant resulted in a delayed onset of clinical signs and viremia onset. ${ }^{75}$ A recombinant silkworm baculovirus, which encodes the P1-2A and 3C protease of FMDV Asia 1, was able to produce specific antibodies in vaccinated animals and protected after challenge with virulent homologous virus, and clinical signs were alleviated and delayed. ${ }^{64}$

A single dose of defective adenovirus 5 (Ad5) containing the $\mathrm{P} 1$ and the $3 \mathrm{C}$ coding region of serotype A FMDV (Ad5A24) was induced, neutralizing antibodies and protecting swine against homologous challenge. ${ }^{76}$ However, the effect on the cellular immune arm was not investigated.

For both vaccinia virus and baculovirus driven expression of empty A-serotype capsids; containing rationally designed mutations, stability was enhanced in eukaryotic cells. $^{77}$ This method to produce vaccinal antigen has a number of prospective benefits over current technologies in terms of production costs, risk of infection, and thermotolerant vaccine. ${ }^{45}$

Human adenovirus type 5 vector expressing capsid protein (P1-2A and mutated viral 3C protease) confer a significant humoral, cellular, and mucosal immunity was elicited in BALB/c mice. Vaccination of guinea pigs elicited substantial neutralizing antibodies and anti-FMDV immunoglobulin A (IgA) antibodies with $100 \%$ protection of guinea pigs against challenge. ${ }^{78}$

A recombinant canine adenovirus type 2 (CAV2) expressing capsid proteins (P1/3C) (Figure 1) was able to trigger a strong humoral immune response in guinea pigs. However, canine adenovirus type 2 (CAV2)-expressing VP1 protein did not elicit a sustaining antibody response in guinea pigs or mice. ${ }^{79}$

Swine vaccinated with Adenovirus 5 with cytomegalovirus enhancer coding A24-2B (Ad5-CI- A24-2BC) established a peak neutralizing antibody response by 7-14 dpv and elicited higher IgM production at $7 \mathrm{dpv}{ }^{14}$ A modified cytomegalovirus promoter enhanced the efficacy of the vector and advancement to increase cell infection in the cell culture, the group receiving the vaccine was completely protected after challenge. ${ }^{14}$

Intramuscular inoculation of mice with recombinants, recombinant capsid protein of FMDV baculovirus cloned with cytomegalovirus immediate early enhancer as a promoter (CMV-IE) and T-cell immunogen coding region with T-cell epitopes, were effectively induced neutralizing antibodies and gamma interferon (IFN- $\gamma$ ). ${ }^{80} \mathrm{P} 1-$ $2 \mathrm{~A}$ and $3 \mathrm{C}$ coding regions FMD serotype $\mathrm{A}$ expressed in silkworm pupae (Bombyx mori) were able to induce high titers of specific antibodies and completely protected against virulent homologous virus challenge. ${ }^{81}$

The multiple antigenic peptide system is highly immunogenic as compared to the single linear peptide of FMD vaccinal antigens. ${ }^{82}$ Synthetic dendrimer peptides, which harbors two copies of the major FMDV antigenic B-cell antigenic site [VP1 (140-158)], covalently linked to a heterotypic T-cell antigenic site from the non-structural protein $3 \mathrm{~A}$ [3A $(21-35)$ ], has been shown to protect pigs against viral challenge. ${ }^{83}$ Dendrimer peptide reproducing the heterotypic and highly conserved FMDV 3A (21-35) T-cell epitope has also improved neutralizing antibody and IFN- $\gamma$ responses. ${ }^{84}$ In $70 \%$ of B2T-vaccinated pigs, full protection - no clinical signs of disease - was observed upon virus challenge at day 25 post-immunization. ${ }^{84}$

\section{DNA Vaccines}

Encephalomyocarditis virus (EMCV) internal ribosome entry site (IRES) has been deleted and L gene, which is involved in cell shutoff by proteolysis of eIF46, ${ }^{85}$ has been removed and the EMCV IRES, which has been shown to increase expression efficiency, has been inserted upstream of the P1 sequences. ${ }^{86}$ DNA vaccine coding P1-2A and GM-CSF as an adjuvant induced robust FMDV specific and neutralizing antibody, as well as indorsing cytokines IL-8 and IFN $\gamma$ production in swine. ${ }^{87}$

DNA vaccines based on viral mini genes corresponding to three major B- and T-cell FMDV epitopes of VP1 (amino acid sequence 133-156)-3A (amino acid sequence 11-40) and VP4 (20-34) protect mice, in the absence of specific antibodies at the time of challenge. ${ }^{88}$

Intranasal administration of the FMDV DNA vaccine; using chitosan as a delivery vehicle and IL-15 as the molecular adjuvant, have induced mucosal and systemic immune response with enhanced cell-mediated immunity (CMI), as shown by the higher level of T-cell proliferation, CTL response, and expression of IFN- $\gamma$ in both $\mathrm{CD}_{4}+$ and $\mathrm{CD}_{8}+$ T-cells. $^{73}$ 
Mice vaccinated with plasmid expressing VP1 and IL9 as a genetic adjuvant and with the anti-apoptosis mechanism triggered, have developed a strong humoral response, high level of IFN- $\gamma$ and perforin in $\mathrm{CD}_{8}+$ T-cells, but not with IL-17 in these T-cells. IL-9 upregulated the expressions of Beclin gene and prevented apoptosis of T-cells. ${ }^{89}$

Another study revealed that VP1 DNA vaccine expressing interleukin- 6 and IFN- $\alpha$ used as molecular adjuvants have improved antigen-specific cell-mediated responses. It also induced a high titer of IgG2a/IgG1, IFN- $\alpha$, IL-4, and dendritic cells maturation. ${ }^{90}$

Using IL-2 as a genetic adjuvant in DNA vaccine encoding, two FMDV VP1 epitopes (amino acid residues 141160 and 200-213) comprising multiple epitopes have to elicit both T-cell proliferation and neutralizing antibody against FMD in swine using IL-2 as a genetic adjuvant. ${ }^{91,92}$

Pigs immunized with the anti-FMDV DNA vaccine plasmid encoding P1-2A3C3D and a plasmid expressing porcine 'B-cell activating factor belonging to the TNF family' (BAFF) promoted B-cell maturation activation and immunoglobulin class switching. ${ }^{93}$

A replicase-based DNA vaccine with regular boosting offered an efficient vaccine strategy against FMDV. ${ }^{94}$ Incorporation of different antigenic targets in DNA vaccines are a perfect way to make an antigen cocktail in a single vaccine formulation. Mice immunized with three plasmids encoding the antigen of foot-and-mouth disease virus (FMDV), pseudorabies virus (PRV), and classic swine fever virus (CSFV) have shown promising results. ${ }^{95}$

Intramuscular inoculation of guinea pigs with DNA plasmids expressing FMDV containing a signal sequence of the swine IgG gene inoculated showed a neutralizing antibody response and spleen cell proliferation increased after boosting, but animals were not protected from viral challenge. ${ }^{96}$

DNA vaccine encoding T-cell epitope and B-cell epitopes from sites 135-167 of VP1 and site 1 includes the 141-160 regions (G-H loop) and the carboxyl terminus of VP1 of FMDV type $\mathrm{O}$ have elicited strong cellular immune response as observed using T-cell proliferation assay. ${ }^{97}$

Intranasal delivery of FMDV DNA vaccine encoding capsid protein, Cationic PLGA (poly(lactide-co-glycolide) as a vehicle and bovine IL-6 as a genetic adjuvant have shown enhanced mucosal and systemic immune responses in vaccinated animals. ${ }^{98}$

DNA vaccine expressing capsid protein (P1-2A, 3C, and 3D) primed with pGM-CSF and boosted with inactivated FMDV antigen showed a substantial level of cross- serotype reactivity in vaccinated pigs. A significant level of cross-serotype reactivity were reported against A, C, and Asial in the virus neutralization and ELISA tests. ${ }^{99}$ However, DNA vaccine expressing VP1 protein and producing antisense RNA targeted to the 5'UTR of FMDV induced a specific immune response in vaccinated mice. ${ }^{72}$

A DNA vaccine expressing VP1 along with IL-15 (molecular adjuvant) boosted mucosal secreted IgA and serum IgG and cell-mediated immunity (CMI), as proven by higher levels of antigen-specific T-cell proliferation, cytotoxic $\mathrm{T}$ lymphocyte (CTL) response, and higher expression of IFN- $\gamma$ in both $\mathrm{CD}_{4}+$ and $\mathrm{CD}_{8}+\mathrm{T}$-cells informing the spleen and mucosal sites. ${ }^{73}$

Recombinant vaccines are made by recombining the host Ig super family and the viral epitopes have improved the humoral and cellular immune response of vaccinated animals. RNA vaccines are potent IgG class switches, in addition to this a high titer of IgM has also been observed in vaccinated mice. ${ }^{100}$ Plasmid DNA containing epitopes of FMDV have ideal tissue distribution in mice. ${ }^{101}$

\section{Challenges in Induction of Long Lasting Immunity}

There is a significant alteration of T-lymphocyte subpopulations, functional competence, and abundance after infection with different serotypes of FMDV. ${ }^{102}$ There is a down-regulation of $\mathrm{boCD}_{4}+$ and $\mathrm{boCD}_{8}+\mathrm{T}$-cells until 48 hours post-infection (hpi). However, down-regulation of boWC1+ T-cells is observed up to 48 hpi with FMDV serotype $\mathrm{O}$. Lymphocytes from vaccinated animals demonstrated a significant up-regulation of $\mathrm{boCD}_{4}+, \mathrm{boCD}_{8}+$, and boWC1+ T-cells following exposure to FMDV. ${ }^{102}$

After natural infection there is a significant increase in 3A-NS protein expression in lymphocytes different at different course of the disease which leads to transient immuno-suppression of $\mathrm{CD}_{4}{ }^{+}$and $\mathrm{CD}_{8}{ }^{+}$T-cells. ${ }^{34}$

Destruction of the host protein trafficking by NS proteins, especially $3 \mathrm{~A}$, disrupts completely, so does not elicit a $\mathrm{CD}_{8}+$ T-cell response, ${ }^{31}$ due to weak CTL the virus persisted in the animal. Integrin receptors mediate bone marrow derived DC apoptosis, hindering the innate immunity of the host. ${ }^{32}$ Another important phenomenon that interferes with host immunity is $\mathrm{Lb}^{\text {pro }}$, a highly conserved domain which plays an important role by inhibiting ubiquitination of key signaling molecules in activation of type I IFN response like retinoic acid-inducible gene I (RIG-I), TANK-binding kinase 1 (TBK1), TNF receptor-associated 
factor 6 (TRAF6), and TRAF3. ${ }^{103}$ This decreases the level of immediate and early initiation of IFN $\beta$ mRNA and IFNstimulated gene products. ${ }^{102}$ Furthermore, $3 \mathrm{C}^{\text {pro }}$ blocks the intra-Golgi transport by degrading protein required for intra-Golgi transport.

Guzman et $\mathrm{al}^{33}$ and Joshi et $\mathrm{al}^{34}$ analyzed surrogate responses for CTL killing, such as proliferation or production of IFN $\gamma$ by $\mathrm{CD}_{8}$ expressing cells which were reported but many CD8+ expressing lymphocytes that are not CTLs which produce IFN $\gamma$, including NK cells and subsets of $\gamma \delta$ T-cells. ${ }^{35-37,39,104}$

The activity of CTL evaluated 10 days after challenge with Ad5-FMDV-3C indicated a significant increase in CTL activity 10 days after challenge compared to preboost levels, but reverted to baseline levels by 17 days after challenge. ${ }^{17}$ However, an attempt to evaluate CTL activity on day 4 failed to obtain enough cells. This was due to lymphopenia and immunopathology induced by FMDV. There is a positive association between IFN- $\gamma$ response and vaccine-induced protection besides a decline of long-term persistence of FMD virus. ${ }^{38}$

According to $\mathrm{Oh}$ et $\mathrm{al},{ }^{38} \mathrm{CD} 4^{+} \mathrm{T}$-cells are the major multiplying phenotype and IFN- $\gamma$ producing cells.

Regardless of FMDV serotype, serum IFN- peaked 2-3 days after infection, lymphopenia corresponded with peak viremia and serum IFN- response, and circulating plasmocyte dendritic cell $(\mathrm{pDC})$ counts and in vitro $\mathrm{pDC}$ IFN- production transiently dropped after 48 hours. Regardless of the FMDV serotype injected or the age of the animal affected, infection of lymphocytes or $\mathrm{pDCs}$ was never found. ${ }^{39}$

The generation of IFN- from monocyte-derived DCs (MoDCs) and skin-derived DCs (skin DCs) is suppressed during the acute phase of swine infection. This impact happens in tandem with increased viral titers in the blood, but these cells are not infected productively. Interestingly, the capacity of these DCs to take up particles and process antigens does not alter, demonstrating that antigens do not affect their ability to take up particles and process antigens. ${ }^{35}$

\section{Conclusion}

Based on the above literature and research gap, it forward the following recommendations. Cellular internalization, glycosylation pattern, antigen presentation, and mechanisms of positive selection (pathogenic strain development) of the traditional vaccines should be studied. Vaccine induced $\mathrm{CD}^{+} \mathrm{T}$ CMI strengthens the long-lived immune response and cross-protection. There is a role of $\delta \gamma$ T-cell receptors in the immune pathogenesis, persistence, and CTL production, which should be studied extensively. Recombinant protein, under the use of flow cytometer, and ELISpot ELISA for the analysis of vaccine particle internalization, antigen presentation, and assessment of antigen presenting cells cross talk. New web-based tools should be developed that will show the effects of side chains on the B- or T-cell epitope. The use of animal model infection and vaccine development and efficacy tests should be sought clearly, because there is a remarkable integrin receptor difference in laboratory animals and swine and ruminants are there. Computational estimation of genome-wide CTL epitopes by integrating epitope conjecturing tools in computing a vast number of viral sequences and subsequent in vivo evaluation have a great advantage to produce vaccines with long-lasting protection and cross-protection capability.

\section{Abbreviations}

3A, Non-structural protein; Ad5, Adenovirus 5 Vector; $3 \mathrm{C}^{\text {pro }}, 3 \mathrm{C}$ protease (Non-structural protein); BEI, Binary Ethylenemines; BHK cell, Baby hamster kidney cell; CD4 + , Cluster differentiation factor four positive; $\mathrm{CD} 8^{+}$, Cluster differentiation factor eight positive; cDNA, Complementary DNA; CTL, Cytotoxic T-cells; DC, Dendritic cell; ELISA, Enzyme linked Immunosorbent assay; FMD, Foot and mouth disease; FMDV, Foot and mouth disease virus; GM-CSF, Granulocytes and Monocytes Colony stimulating factor; IFN- $\alpha$, Interferon alpha; IFN- $\gamma$, Interferon gamma; Ig, Immunoglobulin; IL, Interleukin; LTR, Long terminal repeat; NK cells, Natural killer cells; NS protein, nonstructural protein; P1-2A, Gene for structural protein precursor; P1-2A3C3D, Viral structural protein precursor $\mathrm{P} 1-2 \mathrm{~A}$ and the nonstructural proteins 3C and 3D; PBMC, Peripheral blood mononucleated; RT- PCR, Reverse transcription polymerase chain reaction; SAT, South African Type; TGE, Transient gene expression; TGF, Tumor growth factor; TNF, Tumor necrosis factor; Th cells, T helper cells; VP1, Viral protein 1; $\gamma \delta \mathrm{T}$ cells, Gamma delta T cells.

\section{Data Sharing Statement}

The data used in this review are secondary data and included in the article.

\section{Acknowledgment}

I am very grateful to the Research and Community service vice president office of the University of Gondar for providing materials and financial support. I also thank College 
of Veterinary Medicine and Animal Sciences, University of Gondar, for additional facility support.

\section{Funding}

The author thank University of Gondar, Research and Community service vice president office.

\section{Disclosure}

The author declares that there are no conflicts of interest in this work.

\section{References}

1. Kitching P, Hammond J, Jeggo M, et al. Global FMD control is it an option? Vaccine. 2007;25(30):5660-5664. doi:10.1016/j. vaccine.2006.10.052

2. Raza S, Siddique K, Rabbani M, et al. Microbial pathogenesis in silico analysis of four structural proteins of aphthovirus serotypes revealed significant B and T cell epitopes. Microb Pathog. 2019;128 (August 2018):254-262. doi:10.1016/j.micpath.2019.01.007

3. Arzt J, Belsham GJ. Transmission of foot-and-mouth disease from persistently infected carrier cattle to naive cattle via transfer of oropharyngeal fluid. Vet World. 2018;3:318. doi:10.1128/ mSphere.00365-318

4. Arzt J, Pacheco JM, Stenfeldt C. Pathogenesis of virulent and attenuated foot-and-mouth disease virus in cattle. Vet World. 2017;14:89. doi:10.1186/s12985-017-0758-759

5. Sobhy NM, Bayoumi YH, Mor SK, El-Zahar HI, Goyal SM. Outbreaks of foot and mouth disease in Egypt: molecular epidemiology, evolution and cardiac biomarkers prognostic significance. Int J Vet Sci Med. 2018;6(1):22-30. doi:10.1016/j. ijvsm.2018.02.001

6. Senthilkumaran C, Yang M, Bittner H, et al. Detection of genome, antigen, and antibodies in oral fluids from pigs infected with foot-and-mouth disease virus. Can J Vet Res. 2017;81:82-90.

7. Palinski RM, Bertram MR. First genome sequence of footand-mouth disease virus serotype $\mathrm{O}$ sublineage Ind2001e from Southern Vietnam. Microbiol Resour Announc. 2019;8: e01424-18. doi:10.1128/mra.01424-1418

8. Pulido MR, Sobrino F, Borrego B, et al. Attenuated foot-andmouth disease virus RNA carrying a deletion in the $3^{\prime}$ noncoding region can elicit immunity in swine. $J$ Virol. 2009;83(8):3475-85. doi:10.1128/JVI.01836-08

9. Saravanan P, Iqbal Z, Selvaraj DPR, Aparna M, Umapathi V. Comparison of chemical extraction methods for determination of $146 \mathrm{~S}$ content in foot-and-mouth disease oil-adjuvanted vaccine. J Appl Microbiol. 2019;1(Rueckert 1985):1-9. doi:10.1111/jam.14465

10. Jamal SM, Belsham GJ. Foot-and-mouth disease: past, present and future. Vet Res. 2013;44(1):1-14. doi:10.1186/1297-971644-116

11. Robinson L, Knight-Jones TJ, Charleston B, et al. Global foot-and-mouth disease research update and gap analysis: 7 pathogenesis and molecular biology. Transbound Emerg Dis. 2016;63(1):63-71. doi:10.1111/tbed.12520

12. Sreenivasa BP, Mohapatra JK, Pauszek SJ, et al. Recombinant human adenovirus-5 expressing capsid proteins ofIndian vaccine strains offoot-and-mouth disease virus elicits effective antibody response in cattle. Vet Microbiol. 2017;203:196-201. doi:10.1016/ j.vetmic.2017.03.019
13. Neilan JG, Schutta C, Barrera J, et al. Efficacy of an adenovirus-vectored foot- and-mouth disease virus serotype A subunit vaccine in cattle using a direct contact transmission model. BMC Vet Res. 2018;14(1):1-9. doi:10.1186/s12917-0181582-1

14. Pena L, Pires M, Koster M, et al. Delivery of a foot-and-mouth disease virus empty capsid subunit antigen with nonstructural protein 2B improves protection of swine. Vaccine. 2008;26 (45):5689-5699. doi:10.1016/j.vaccine.2008.08.022

15. Barteling SJ, Cassim NI. Very fast (and safe) inactivation of footand-mouth disease virus and enteroviruses by a combination of binary ethyleneimine and formaldehyde. Dev Biol (Basel). 2004;119:449-455

16. Rweyemamu MM, Umehara O, Giorgi W, Medeiros R, Neto DL, Baltazar M. Effect of formaldehyde and binary ethyleneimine (BEI) on the integrity of foot and mouth disease virus capsid. Rev Sci Tech. 1989;8(3):747-764. doi:10.20506/rst.8.3.425

17. Patch JR, Kenney M, Pacheco JM, Grubman MJ, Golde WT. Characterization of cytotoxic $\mathrm{T}$ lymphocyte function after foot-and-mouth disease virus infection and vaccination. Viral Immunol. 2013;26(4):239-249. doi:10.1089/vim.2013.0011

18. Rodriguez-Calvo T, Ojosnegros S, Sanz-ramos M, Garcia-Arriaza J, Escarmis C, Domingo E, Sevilla N. New vaccine design based on defective genomes that combines features of attenuated and inactivated vaccines. PLoS One. 2010;5(4):1-11. doi:10.1371/ journal.pone.0010414

19. Núñez JI, Baranowski E, Molina N, et al. Acid substitution in nonstructural protein $3 \mathrm{~A}$ can mediate adaptation of foot-andmouth disease virus to the Guinea pig. J Virol. 2001. doi:10.1128/JVI.75.8.3977-3983.2001

20. Burman A, Clark S, Abrescia NGA, et al. Specificity of the VP1 $\mathrm{GH}$ loop of foot-and-mouth disease virus for $\alpha \mathrm{v} \beta 6$ integrins. J Virol. 2006;80(19):9798-9810. doi:10.1128/JVI.00577-06

21. Dicara D, Burman A, Clark S, et al. Foot-and-mouth disease virus forms a highly stable, EDTA-resistant complex with its principal receptor, integrin $\alpha v \beta 6$ : implications for infectiousness. $J$ Virol. 2008;82(3):1537-1546. doi:10.1128/JVI.01480-07

22. Domingo E, Sheldon J, Perales C, et al. Viral quasispecies evolution. Microbiol Mol Biol Rev. 2012;76(2):159-216. doi:10.1128/MMBR.05023-11

23. Mckenna TS, Lubroth J, Rieder E, et al. Receptor binding site-deleted foot-and-mouth disease (FMD) virus protects cattle from FMD. $J$ Virol. 1995;69(9):5787-5790. doi:10.1128/ jvi.69.9.5787-5790.1995

24. Dimarchi R, Brooke G, Gale C, Cracknell V, Doel T, Mowat N. Protection of Cattle Against Foot-and-Mouth Disease by a Synthetic Peptide. Science. 1986; 232:639-641. doi:10.1126/science.3008333

25. Gómez N, Salinas J, Escribano JM, et al. Protective immune response to foot-and-mouth disease virus with VP1 expressed in transgenic plants protective immune response to foot-and-mouth disease virus with VP1 expressed in transgenic plants. J Virol. 1998;72:2-5.

26. Botton SDA, Brum MCS, Bautista E, et al. Immunopotentiation of a foot-and-mouth disease virus subunit vaccine by interferon alpha. Vaccine. 2006;24(17):3446-3456. doi:10.1016/j. vaccine.2006.02.011

27. Shi X, Wang B, Wang M. Immune enhancing effects of recombinant bovine IL-18 on foot-and-mouth disease vaccination in mice model. Vaccine. 2007;25(7):1257-1264. doi:10.1016/j.vaccine.2006.10.017

28. Wang F, He X, Jiang L, et al. Enhanced immunogenicity of microencapsulated multiepitope DNA vaccine encoding $\mathrm{T}$ and $\mathrm{B}$ cell epitopes of foot-and-mouth disease virus in mice. Vaccine. 2017;24(2006):2017-2026. doi:10.1016/j. vaccine.2005.11.042 
29. Wan Y, Ren X, Ren Y, et al. As a genetic adjuvant, CTA improves the immunogenicity of DNA vaccines in an ADP-ribosyltransferase activity- and IL-6-dependent manner. Vaccine. 2014;32(19):2173-2180. doi:10.1016/j.vaccine.20 14.02.056

30. Lee MJ, Jo H, Shin SH, Kim S, Kim B, Park J. Mincle and STING-stimulating adjuvants elicit robust cellular immunity and drive long-lasting memory responses in a foot-and-mouth disease vaccine. Front Immunol. 2019;10(October):1-14. doi:10.3389/ fimmu.2019.02509

31. Cornell CT, Kiosses WB, Whitton JL, et al. Inhibition of protein trafficking by coxsackievirus B3: multiple viral proteins target a single organelle. J Virol. 2006;80(13):6637-6647. doi:10.1128/ JVI.02572-05

32. Jin $\mathrm{H}$, Xiao $\mathrm{C}$, Zhao $\mathrm{G}$, et al. Induction of immature dendritic cell apoptosis by foot and mouth disease virus is an integrin receptor mediated event before viral infection. J Cell Biochem. 2007;991 (4):980-991. doi:10.1002/jcb.21332

33. Guzman E, Taylor G, Charleston B, et al. Induction of a cross-reactive CD8+ $\mathrm{T}$ cell response following foot-and-mouth disease virus vaccination. J Virol. 2010;84(23):12375-12384 doi:10.1128/JVI.01545-10

34. Joshi G, Sharma R, Kakker NK. Phenotypic and functional characterization of T-cells and in vitro replication of FMDV serotypes in bovine lymphocytes. Vaccine. 2009;27(48):6656-6661. doi:10.1016/j.vaccine.2009.08.107

35. Nfon CK, Ferman GS, Toka FN, Gregg DA, Golde WT. Interferon- production by swine dendritic cells is inhibited. Viral Immunol. 2008;21(1):68-77. doi:10.1089/vim.2007.0097

36. Golde WT, Nfon CK, Toka FN. Immune evasion during foot-andmouth disease virus infection of swine. Immunol Rev. 2008;225 (85-89):85-95. doi:10.1111/j.1600-065X.2008.00672.x

37. Toka FN, Nfon C, Dawson H, Golde WT. Natural killer cell dysfunction during acute infection with foot-and-mouth disease virus. Clin Vaccine Immunol. 2009;16(12):1738-1749. doi:10.112 8/CVI.00280-09

38. Oh Y, Fleming L, Statham B, et al. Interferon- $\mathrm{c}$ induced by in vitro re-stimulation of $\mathrm{CD} 4+\mathrm{T}$-cells correlates with in vivo FMD vaccine induced protection of cattle against disease and persistent infection. PLoS One. 2012;7(9). doi:10.1371/journal. pone. 0044365

39. Nfon CK, Toka FN, Kenney M, Pacheco JM, Golde WT. Loss of plasmacytoid dendritic cell function coincides with lymphopenia and viremia duringFoot Mouth Dis Virus infection. Viral Immunity. 2010;23(1):29-41. doi:10.1089/vim.2009.0078

40. Reid E, Juleff N, Gubbins S, et al. Bovine plasmacytoid dendritic cells are the major source of type I interferon in response to foot-and-mouth disease virus in vitro and in vivo. $J$ Virol. 2011;85(9):4297. doi:10.1128/JVI.02495-10

41. Zhao G, Jin H, Li J, et al. PyNTTTTGT prototype oligonucleotide IMT504, a novel effective adjuvant of the FMDV DNA vaccine. VIRAL Immunol. 2009;22(2):131-138. doi:10.1089/vim.200 8.0073

42. Doel TR. Review FMD v accines. Vaccine. 2003;91:81-99.

43. Carrillo C, Lu Z, Borca MV, Vagnozzi A, Kutish GF, Rock DL. Genetic and phenotypic variation of foot-and-mouth disease virus during serial passages in a natural host. $J$ Virol. 2007;81 (20):11341-11351. doi:10.1128/JVI.00930-07

44. Mateo R, Luna E, Mateu MG, Mateu MG. Engineering viable foot-and-mouth disease viruses with increased thermostability as a step in the development of improved vaccines. J Virol. 2008;82 (24):12232-12240. doi:10.1128/JVI.01553-08

45. Rodriguez LL, Grubman MJ. Foot and mouth disease virus vaccines. Vaccine. 2009;27:90-94. doi:10.1016/j.vaccine.200 9.08.039
46. Gladue DP, Donnell VO, Baker-branstetter R, et al. Foot-andmouth disease virus nonstructural protein $2 \mathrm{C}$ interacts with Beclin1, modulating virus replication. $J$ Virol. 2012;86 (22):12080-12090. doi:10.1128/JVI.01610-12

47. Núñez JI, Baranowski E, Molina N, Ruiz-jarabo CM, Sánchez C, Sobrino F. A single amino acid substitution in nonstructural protein $3 \mathrm{~A}$ can mediate adaptation of foot-and-mouth disease virus to the Guinea pig. $J$ Virol. 2001;75(8):3977-3983. doi:10.1128/JVI.75.8.3977

48. Patil PK, Suryanarayana V, Bist P, Bayry J, Natarajan C. Integrity of GH-loop of foot-and-mouth disease virus during virus inactivation: detection by epitope specific antibodies. Vaccine. 2002;20 (7-8):1163-1168. doi:10.1016/S0264-410X(01)00431-5

49. Rweyemamu MM, Umenera O, Giorgi W, Medeiros R, Neto DL, Blatazar M. Effect of formaldehyde and binary ethyleleimine (BEI) on the integrity of foot and mouth virus capsid. Rev Sci Tech. 1989;8(3):747-764.

50. Martín-acebes MA, Vázquez-calvo Á, González-magaldi M, Sobrino F. Foot-and-mouth disease virus particles inactivated with binary ethylenimine are efficiently internalized into cultured cells. Vaccine. 2011;29(52):9655-9662. doi:10.1016/j.vaccine.20 11.10.031

51. Patil PK, Bayry J, Ramakrishna C, Hugar B, Misra LD, Natarajan C. Immune responses of goats against foot-and-mouth disease quadrivalent vaccine: comparison of double oil emulsion and aluminium hydroxide gel vaccines in eliciting immunity. Vaccine. 2002;20(21-22):2781-2789. doi:10.1016/S0264-410X (02)00184-6

52. Gebauer F, Gomes I, Mateu MG, et al. Rapid selection of genetic and antigenic variants of foot-and-mouth disease virus during persistence in cattlet. $J$ Virol. 1988;62(6):2041-2049. doi:10.1128/jvi.62.6.2041-2049.1988

53. Martín-acebes MA, Vázquez-calvo Á, Mateu MG, et al. Acid substitution in the capsid of foot-and-mouth disease virus can increase acid resistance. $J$ Virol. 2011;85(6):2733-2740. doi:10.1128/JVI.02245-10

54. Jackson T, Sheppard D, Denyer M, Blakemore W, King AMQ. The epithelial integrin $\alpha v \beta 6$ is a receptor for foot-and-mouth disease virus. J Virol. 2000;74(11):4949-4956. doi:10.1128/ JVI.74.11.4949-4956.2000

55. Harwood LJ, Gerber H, Sobrino F, Summerfield A, Mccullough KC, Irol JV. Dendritic cell internalization of foot-and-mouth disease virus: influence of heparan sulfate binding on virus uptake and induction of the immune response. J Virol. 2008;82(13):6379-6394. doi:10.1128/JVI.00021-08

56. Tami C, Taboga O, Berinstein A, et al. Evidence of the coevolution of antigenicity and host cell tropism of foot-and-mouth disease virus in vivo. J Virol. 2003;77(2):1219-1226. doi:10.1128/ JVI.77.2.1219

57. Juleff ND, Maree FF, Waters R, Bengis RG, Charleston B. The importance of FMDV localisation in lymphoid tissue. Vet Immunol Immunopathol. 2012;148(1-2):145-148. doi:10.1016/j. vetimm.2011.05.013

58. Pulido MR, Sobrino F, Borrego B, et al. Attenuated Foot-andmouth disease virus RNA carrying a deletion in the $3^{\prime}$ noncoding region can elicit immunity in swine. $J$ Virol. 2009;83 (8):3475-3485. doi:10.1128/JVI.01836-08

59. Cedillo-barron L, Foster-cuevas M, Cook A, et al. Immunogenicity of plasmids encoding $\mathrm{T}$ and $\mathrm{B}$ cell epitopes of foot-and-mouth disease virus (FMDV) in swine. Vaccine. 2003;21 (27-30):4261-4269. doi:10.1016/S0264-410X(03)00453-5

60. Li P, Bai X, Sun P, et al. Evaluation of a genetically modified foot-and-mouth disease virus vaccine candidate generated by reverse genetics. BMC Vet Res. 2012;8(1):1. doi:10.1186/1746$6148-8-57$ 
61. Barnett PV, Cox SJ, Aggarwal N, Gerber H, Mccullough KC. Further studies on the early protective responses of pigs following immunisation with high potency foot and mouth disease vaccine. Vaccine. 2002;20(25-26):3197-3208. doi:10.1016/S0264-410X (02)00242-6

62. Yadav S, Sharma R, Chhabra R. Interleukin-2 potentiates foot-and-mouth disease vaccinal immune responses in mice. Vaccine. 2005;23:3005-3009. doi:10.1016/j.vaccine.2004.12.005

63. Cox SJ, Aggarwal N, Statham RJ, Barnett PV. Longevity of antibody and cytokine responses following vaccination with high potency emergency FMD vaccines. Vaccine. 2003;21(1314):1336-1347. doi:10.1016/S0264-410X(02)00691-6

64. Li Z, Yi Y, Yin X, Zhang Z, Liu J. Expression of foot-and-mouth disease virus capsid proteins in silkworm-baculovirus expression system and its utilization as a subunit vaccine. PLoS One. 2008;3 (5):1-7. doi:10.1371/journal.pone.0002273

65. Grubman MJ, Morgan DO, Kendall J, Baxt B. Capsid intermediates assembled in a foot-and-mouth disease virus genome RNA programmed cell-free translation system and in infected cells. J Virol. 1985;56(1):120-126. doi:10.1128/jvi.56.1.120-126.1985

66. Mignaqui AC, Ruiz V, Perret S, et al. Transient gene expression in serum-free suspension-growing mammalian cells for the production of foot-and- mouth disease virus empty capsids. PLoS One. 2013;8(8):1-9. doi:10.1371/journal.pone.0072800

67. Höhlich B, Wiesmüller K, Schlapp T, et al. Identification of foot-and-mouth disease virus-specific linear B-cell epitopes to differentiate between infected and vaccinated cattle. $J$ Virol. 2003;77(16):8633-8639. doi:10.1128/JVI.77.16.8633

68. Perkins J, Parida S, Clavijo A, Perkins J, Parida S, Clavijo A. Use of a standardized bovine serum panel to evaluate a multiplexed nonstructural protein antibody assay for serological surveillance of foot-and-mouth disease. $J$ Virol. 2007;14(11):1472-82. doi:10.1128/CVI.00227-07

69. Ko Y, Lee H, Jeoung H, et al. Use of a baculovirus-expressed structural protein for the detection of antibodies to foot-andmouth disease virus type A by a blocking enzyme-linked immunosorbent assay. Clin Vaccine Immunol. 2010;17(1):194-198. doi:10.1128/CVI.00374-09

70. Zhang L, Zhang J, Chen H, Zhou J, Ding Y, Liu Y. Research in advance for FMD novel vaccines. Virol J. 2011;8(268):1-6. doi:10.1186/1743-422X-8-268

71. Alexandersen S, Zhang Z, Donaldson AI, Garland AJM. The pathogenesis and diagnosis of foot-and-mouth disease. J Comp Path. 2003;129(3):1-36. doi:10.1016/S0021-9975(03)00041-0

72. Yang B, Lan X, Li X, et al. A novel bi-functional DNA vaccine expressing VP1 protein and producing antisense RNA targeted to 5 UTR of foot-and-mouth disease virus can induce both rapid inhibitory effect and specific immune response in mice. Vaccine. 2008;26(43):5477-5483. doi:10.1016/j.vaccine.2008.07.060

73. Wang X, Zhang X, Kang Y, et al. Interleukin-15 enhance DNA vaccine elicited mucosal and systemic immunity against foot and mouth disease virus. Vaccine. 2008;26(40):5135-5144. doi:10.1016/j.vaccine.2008.03.088

74. Wang J, Liang C, Peng J, et al. Induction of immunity in swine by purified recombinant VP1 of foot-and-mouth disease virus. Vaccine. 2003;21(25-26):3721-3729. doi:10.1016/S0264-410X(03)00363-3

75. Bracci L, Canini I, Venditti M, et al. Type I IFN as a vaccine adjuvant for both systemic and mucosal vaccination against influenza virus. Vaccine. 2006;2:2005-2006. doi:10.1016/j. vaccine.2005.01.121

76. Moraes MP, Mayr GA, Mason PW, Grubman MJ. Early protection against homologous challenge after a single dose of replication-defective human adenovirus type 5 expressing capsid proteins of foot-and-mouth disease virus (FMDV) strain A24. Vaccine. 2002;20(11-12):1631-1639. doi:10.1016/S0264-410X (01)00483-2
77. Porta C, Kotecha A, Burman A, et al. Rational engineering of recombinant picornavirus capsids to produce safe, protective vaccine antigen. PLoS Pathog. 2013;9(3):e1003255. doi:10.1371/ journal.ppat. 1003255

78. Xie Y, Chang H, Li Z, Zhang Y. Adenovirus-vectored capsid proteins of the serotype a foot-and-mouth disease virus protect guinea pigs against challenge. Front Microbiol. 2020;11 (July):1-9. doi:10.3389/fmicb.2020.01449

79. De Vleeschauwer AR, Zhou X, Lefebvre DJ, et al. A canine adenovirus type 2 vaccine vector confers protection against foot-and-mouth disease in guinea pigs. Vaccine. 2018;36 (16):2193-2198. doi:10.1016/j.vaccine.2018.02.074

80. Cao Y, Lu Z, Sun P, et al. A pseudotype baculovirus expressing the capsid protein of foot-and-mouth disease virus and a T-cell immunogen shows enhanced immunogenicity in mice. Virol $J$. 2011;8(1):77. doi:10.1186/1743-422X-8-77

81. Li Z, Yin X, Yi Y, et al. FMD subunit vaccine produced using a silkworm - baculovirus expression system: protective efficacy against two type Asia1 isolates in cattle. Vet Microbiol. 2011;149 (1-2):99-103. doi:10.1016/j.vetmic.2010.10.022

82. Forner M, Cañas-arranz R, Defaus S, et al. Peptide-based vaccines: foot-and-mouth disease virus, a paradigm in animal health. Vaccines. 2021;9(5):1-21. doi:10.3390/vaccines 9050477

83. Cañas-Arranz R, de León P, Forner M. Immunogenicity of a dendrimer B $2 \mathrm{~T}$ peptide harboring a T-cell epitope from FMDV non-structural protein. Front Vet Sci. 2020;7 (August):1-10. doi:10.3389/fvets.2020.00498

84. Cañas-arranz R, Forner M, Defaus S, et al. A single dose of dendrimer B2T peptide vaccine partially protects pigs against foot-and-mouth disease virus infection. Vaccines. 2020;8 (19):1-11. doi:10.3390/vaccines8010019

85. Chang Y, Dou Y, Bao H, et al. Multiple microRNAs targeted to internal ribosome entry site against foot-and-mouth disease virus infection in vitro and in vivo. Virol $J$. 2013;11(1):1-12. doi:10.1186/1743-422X-11-1

86. Benvenisti L, Rogel A, Kuznetzova L, Bujanover S, Becker Y, Stram Y. Gene gun-mediate DNA vaccination against foot-andmouth disease virus. Vaccine. 2001;19(28-29):3885-3895. doi:10.1016/S0264-410X(01)00125-6

87. Li Y, Aggarwal N, Takamatsu H, Sterling CMA, Voyce C, Barnett PV. Enhancing immune responses against a plasmid DNA vaccine encoding a FMDV empty capsid from serotype $\mathrm{O}$. Vaccine. 2006;24(21):4602-4606. doi:10.1016/j.vaccine.20 05.08.032

88. Borrego B, Fernandez-pacheco P, Ganges L, Domenech N. DNA vaccines expressing $\mathrm{B}$ and $\mathrm{T}$ cell epitopes can protect mice from FMDV infection in the absence of specific humoral responses. Vacine. 2006;24(18):3889-3899. doi:10.1016/j.vaccine.20 06.02 .028

89. Zou Q, Wu B, He X, et al. Increasing a robust antigen-specific cytotoxic $\mathrm{T}$ lymphocyte response by FMDV DNA vaccination with IL-9 expressing construct. $J$ Biomed Biotechnol. 2010;2010:1-8. doi:10.1155/2010/562356

90. Su B, Wang J, Wang X, et al. The effects of IL-6 and TNF- $\alpha$ as molecular adjuvants on immune responses to FMDV and maturation of dendritic cells by DNA vaccination. Vaccine. 2008;26 (40):5111-5122. doi:10.1016/j.vaccine.2008.03.089

91. Zhang H, Sun S, Guo Y, et al. Optimization strategy for plasmid DNAs containing multiple-epitopes of foot-and-mouth disease virus by cis-expression with IL-2. Vaccine. 2008;26(6):769-777. doi:10.1016/j.vaccine.2007.11.093

92. Wong H, Cheng SC, Sin FW, Chan EW, Sheng Z, Xie Y. A DNA vaccine against foot-and-mouth disease elicits an immune response in swine which is enhanced by co-administration with interleukin-2. Vaccine. 2002;20(21-22):2641-2647. doi:10.1016/ S0264-410X(02)00212-8 
93. Bergamin F, Saurer L, Neuhaus V, Mccullough KC, Summerfield A. Porcine B-cell activating factor promotes anti-FMDV antibodies in vitro but not in vivo after DNA vaccination of pigs. Vet Immunol Immunopathol. 2007;120(34):115-123. doi:10.1016/j.vetimm.2007.06.021

94. Dar PA, Suryanaryana VS, Nagarajan G, Reddy GR, Dechamma HJ, Kondabattula G. DNA prime-protein boost strategy with replicase-based DNA vaccine against foot-and-mouth disease in bovine calves. Vet Microbiol. 2013;163(1-2):62-70. doi:10.1016/j.vetmic.2012.12.017

95. Wang K, Guo Y, Zhang Y, Lv K, Sun S. Combined DNA vaccination against three animal viruses elicits decreased immunogenicity of a single plasmid in mice. Vaccine. 2007;25 (22):4429-4436. doi:10.1016/j.vaccine.2007.03.023

96. Huang H, Yang Z, Xu Q, et al. Recombinant fusion protein and DNA vaccines against foot and mouth disease virus infection in foot-andmouth. Viral Immunol. 1999;12(1):1-8. doi:10.1089/vim.1999.12.1

97. Zhang H, Sun S, Guo Y, et al. Immune response in mice inoculated with plasmid DNAs containing multiple-epitopes of foot-and-mouth disease virus. Vaccine. 2003;21(32):4704-4707. doi:10.1016/S0264-410X(03)00519-X

98. Zhou P, Wang G, Pan L, et al. Intranasal delivery of cationic PLGA nano/microparticles- loaded FMDV DNA vaccine encoding IL-6 elicited protective immunity against FMDV challenge. PLoS One. 2011;6(11):e27605. doi:10.1371/journal.pone.0027605

99. Li Y, Stirling CMA, Denyer MS, et al. Dramatic improvement in FMD DNA vaccine efficacy and cross-serotype antibody induction in pigs following a protein boost. Vaccine. 2008;26 (21):2647-2656. doi:10.1016/j.vaccine.2008.01.037
100. Borrego B, Rodríguez-pulido M, Mateos F, De N, Sobrino F, Sáiz M. Delivery of synthetic RNA can enhance the immunogenicity of vaccines against foot-and-mouth disease virus (FMDV) in mice. Vaccine. 2013;31(40):4375-4381. doi:10.1016/j.vaccine.2013.07.008

101. Zhang H, Sun S, Guo Y, et al. Tissue distribution of a plasmid DNA containing epitopes of foot-and-mouth disease virus in mice. Vaccine. 2005;23(48-49):5632-5640. doi:10.1016/j. vaccine.2005.06.029

102. Santos TDL, Botton SDA, Weiblen R, et al. The leader proteinase of foot-and-mouth disease virus inhibits the induction of beta interferon mRNA and blocks the host innate immune response. J Virol. 2006;80(4):1906-1914. doi:10.1128/JVI.80.4.1906

103. Wang D, Fang L, Li P, et al. The leader proteinase of foot-andmouth disease virus negatively regulates the type I interferon pathway by acting as a viral deubiquitinase. J Virol. 2011;85 (8):3758-3766. doi:10.1128/JVI.02589-10

104. Patch JR, Pedersen LE, Toka FN, et al. Induction of foot-andmouth disease virus-specific cytotoxic $\mathrm{T}$ cell killing by vaccination. Clin Vaccine Immunol. 2011;18(2):280-288. doi:10.1128/CVI.00417-10

105. Belsham, GJ. Towards improvement in foot-and-mouth disease vaccine performance. Acta Vet Scand. 2020;62(1):20. doi:10.1186/s13028-020-00519-1

\section{Publish your work in this journal}

Veterinary Medicine: Research and Reports is an international, peerreviewed, open access journal publishing original research, case reports, editorials, reviews and commentaries on all areas of veterinary medicine. The manuscript management system is completely online and includes a very quick and fair peer-review system. Visit http://www.dovepress.com/testimonials.php to read real quotes from published authors. 